\title{
Investigation of spacial clustering of rare diseases: childhood malignancies in North Humberside
}

\author{
Freda Alexander, Ray Cartwright, Patricia A McKinney, T J Ricketts
}

\begin{abstract}
Study objective-The aims of the study were (1) to test for uniformity of distribution of childhood leukaemias and other malignancies; and (2) to consider the aetiological implications of unusual distributions.
\end{abstract}

Design-A test for spacial clustering was applied using a method which allows for unequal distribution of the population at risk and avoids using census data to provide population denominators. When clustering was identified, four possible aetiological links which had already been suggested to the Leukaemia Research Fund Centre were examined in a local area.

Setting-The study was carried out in the Yorkshire Health Region in the north of England.

Patients-144 children under 15 years of age with a diagnosis of malignant disease known to the Yorkshire Regional Childhood Tumour Registry between 1974 and 1986 were included in the analysis. Of these 53 had leukaemias and nine had lymphomas.

Measurements and results-Significant localised clustering was found in North Humberside, though not in the whole of the Yorkshire Health Region. A number of clustered cases were identified, some of whom were in a post code sector, Hull 10, to the west of Kingston-upon-Hull, about which concern had been expressed since 1985. There was however no evidence that disease clustering was confined to this area. Four previously suggested hypotheses about causation in this particular area were examined but the results were negative or inconclusive.

Conclusions-The identification of spacial clustering must be seen as only the first step in a series of investigations; it can only rarely lead to aetiological conclusions by itself, but it can motivate and target other investigations.

Leukaemia Research Fund Centre for Clinical

Epidemiology, 17 Springfield Mount, Leeds LS2 9NG

F Alexander

R Cartwright

P A McKinney

T J Ricketts

Correspondence to:

Dr Alexander

Accepted for publication June 1989 cancers was noted through reports from a number of different organisations and individuals: the
Early in 1985 the attention of the Yorkshire Regional Children's Tumour Registry was area comprising Anlaby, Willerby and These dormitory villages have a single post code, HU10, but are divided into different electoral wards (28KNAA, KNAG, KNAU, KNAZ). The unusual occurrence of both childhood and adult district medical officer for East Yorkshire, the parent of a child with leukaemia, the headmistress of a local school, and local clinicians all contacted one of us (RAC) with a variety of case descriptions. In addition and more recently the Leukaemia Research Fund (LRF) data collection survey ${ }^{1}$ independently noted an unusual distribution of childhood leukaemias in the area. Various explanations were suggested by the local residents, including attendance at particular schools and possible use of a single classroom, emissions from the stack at the Capper Pass tin smelter, and exposure to dust from the Melton Whiting Works. A preliminary report by two investigators (RAC, PAM) ${ }^{2}$ in July 1985 concluded that there was no significant excess apparent at that time but that the situation should be monitored. Recently, though after the present analysis was completed, the media have noted the "cluster".

Over many years there have been sporadic reports from the United Kingdom, the USA and elsewhere of "clusters" or "micro epidemics" of leukaemia in both adults and children, which have been described in detail in published reviews. ${ }^{34}$ Until as recently as 1978 the motivation for the study of case clustering came almost entirely from the possibility, supported by animal epidemiology, that the disease might have an infectious aetiology. ${ }^{3}$ More recently public interest and even research interest has concentrated more on their relationship with localised exposure to radiation ${ }^{56}$ or chemicals. ${ }^{7}$

In the past the method of choice for statistical analysis was investigation of space-time interaction. ${ }^{8}$ This method is appropriate of investigating epidemics of ordinary infectious diseases but is less suitable for disease with longer and unknown latent period. ${ }^{9}$

There has been intense interest in the last few years in the statistical methodology of investigating spatial clustering. Several new methods have been applied to different data sets by different investigators. One of us (FEA) planned a study to compare these methodologies in their application to indentical data sets, and of those held at the Leukaemia Research Fund centre the most appropriate, because of its long time span, was the Yorkshire Regional Childhood Tumour Registry. A specific aim of the statistical study was to examine robustness to choice of reference area; thus data from a suitable subarea were also required; North Humberside was selected on the grounds that it contained Hull, which was already of interest to the Leukaemia Research Fund but yet was a large and natural area of which the relevant part of Hull was only a very small part. The statistical study is nearly 
complete and will be submitted for publication. Our experience of applying a new method of analysis ${ }^{10}$ to North Humberside and the results obtained are reported now because they are of independent interest, both with respect to the method and as a public health issue. A preliminary report has already been published. ${ }^{11}$

This study is in three parts. The first part, which we refer to as the spatial analysis, is the testing for uniformity of the disease intensity in North Humberside. Until this was completed the statistician (FEA) remained deliberately unaware of the details (location, time period, age range and diagnostic criteria) of the reported "cluster". A priori choices of diagnostic groupings were leukaemias and non-leukaemias, as well as the entire group of childhood malignancies. The decision to consider the leukaemias separately was made because they form the largest single group of childhood malignancies and because it is often suspected that they do form clusters. In the second part of the study we discuss aetiological interpretations, and in the third part we reassess the disease incidence in the area of Hull 10.

\section{Methods}

The Yorkshire Regional Childhood Tumour Registry registers children under 15 years of age diagnosed with malignant disease and certain benign conditions and resident in the Yorkshire Health Region at the time of diagnosis; optimal ascertainment has been attempted for cases diagnosed on or after January 1st 1974. The area of study is the four health districts of Beverley, Kingston-upon-Hull, Holderness and East Yorkshire, as shown in fig 1 . All diagnoses are classified and grouped in a standard fashion. Cases known to the registry from 1972 to the present are reported here, but formal analysis is restricted to cases diagnosed from 1974-1986. Ascertainment was incomplete for 1972 and 1973, and cases diagnosed after 1986 may still be accruing. There were 144 cases: of these 53 were leukaemias and nine were lymphomas. The leukaemias comprised the range of acute and chronic myeloid and lymphoblastic disease, as defined by Birch and Marsden. ${ }^{12}$

The study begins with a "spatial analysis" in which we test for spatial clustering using the method of Cuzick and Edwards. ${ }^{10}$ The method allows for unequal distribution of the population at risk and also avoids using census data to provide population denominators. A sample of control addresses required by the method was obtained by random selection from all births in the four health districts on 7th January and 7th June for each of the years 1974-1986. One hundred and forty one control addresses were selected. If the geographical distribution of births was different from that of the childhood population then the results could be biased. The most appropriate way of testing for this would be to aggregate births by ward over the period 1974-1981 and compare the ward distributions of total births and resident children according to the 1981 census. Unfortunately ward vital statistics do not exist prior to 1981 . We have obtained birth data for 1981 to 1986 (OPCS: SD30) but these use current

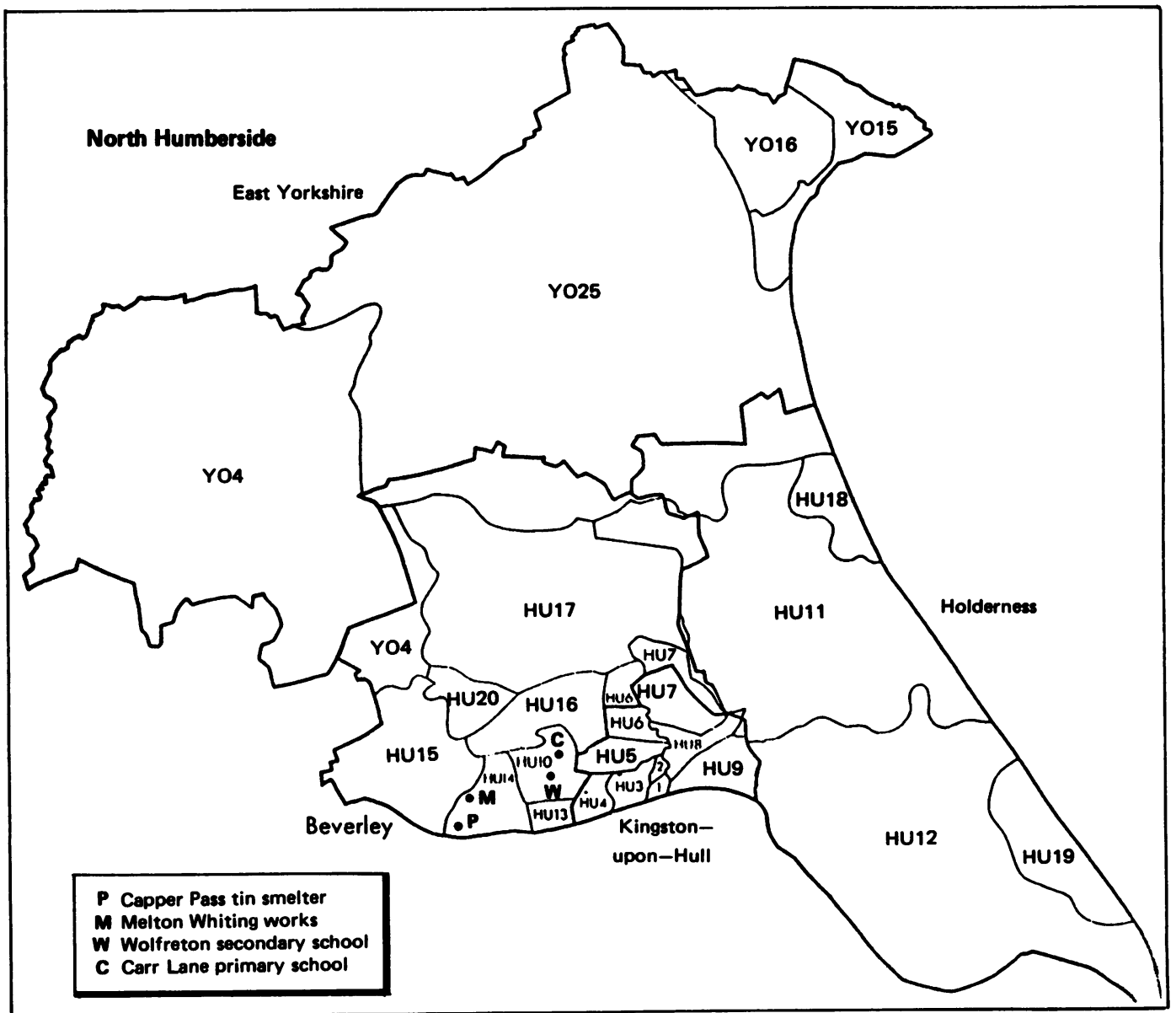


ward boundaries rather than boundaries frozen to the 1981 census. The ward boundaries showed substantial change after 1983. It also shows evidence of considerable annual fluctuation in certain wards so that a comparison of, for example, 1981 births with 1981 census population is probably not appropriate, though it is of interest that the correlation coefficient for total births 1981-1983 against the $0-14$ year population was 0.94 . An alternative justification of the control data is derived from the statistical study mentioned above which used the present control data as a comparison data set. Seven different methods for investigating clustering and heterogeneity were applied to the control data and none found any evidence that the distribution was not uniform. We acknowledge that the choice of controls for the Cuzick-Edwards methods is critical and that our approach is not necessarily ideal. It is, however, difficult to think of a better solution.

The statistical method involves representing all locations of cases and controls, without distinguishing between them, as points on a graph ("graph" is used here in the theoretical sense of a collection of points with lines joining certain pairs). Then for each point lines are drawn to the points representing the three nearest locations. Certain numbers other than three are possible and were considered, but three was found to be most suitable; appropriate adjustments for multiple testing have been made. After this process has been completed case locations are identified and the numbers of lines joining two cases are counted. This is "Cuzick's T3 Statistic"; higher observed than expected values of $\mathrm{T} 3$ indicate spatial clustering of the cases. It is shown in ${ }^{10}$ that T3 has a distribution which is asymptotically normal and formulas for the mean and variance are provided. The results reported here use the normal approximation but the significance levels have been confirmed using 999 simulations with case status allocated randomly to the address locations.

The test investigates a global tendency to cluster, and the authors do not discuss the question of identifying individual clusters. However a natural extension is to select those cases which contribute most to a significant value of T3 and refer to these influential cases as "clustered". Display facilities are not included in the method either. However we have developed a suitable method, details of which are given in the appendix.

The uniformity of the disease intensity is then examined briefly using census wards and Poisson distribution for each. This is a more traditional method and was used, for example, by Gardner and Winter. ${ }^{13}$

The second part of the study begins with the recognition that there is evidence of unusual spatial clustering in North Humberside. In order to seek hypotheses and to examine and illustrate the difficulties inherent in making even tentative aetiological conclusions, we concentrated on Hull 10.

Four potential associations which had already been suggested to the LRF Centre by the community were investigated. These were: (1) attendance at Wolfreton Secondary School; (2) attendance at Carr Lane Primary School; (3) proximity to Capper Pass Factory; (4) proximity to Blue Circle Cement, Melton Whiting. The methods of examining the putative aetiological links are all based on the use of Poisson probabilities to compare observed and expected incidence over areas consisting of combinations of enumeration districts or wards. Expected values were computed using Yorkshire Regional Childhood Tumour Registry rates and 1981 census populations.

The Office of Population Censuses and Surveys (OPCS) post code directory provides for each individual post code unique Ordnance Survey grid references and a census ward so that cases can be assigned unambiguously to wards, though not (in England and Wales) to enumeration districts. However, the Leukaemia Research Fund Centre has developed standard software which allocates each post code to that enumeration district whose centroid ${ }^{\star}$ is nearest to the Ordnance Survey grid reference point (with random selection when there are ties). Consequently each case can also be "assigned" to a unique enumeration district, though this process is not exact.

For the two schools we required estimates of the population denominators for their catchment areas. In the case of Wolfreton Secondary School every enumeration district was tested to see whether its centroid was inside the catchment area of the school. This process yielded an estimate of the catchment area which comprised four wards (with OPCS Small Area Statistics SAS codes 28KNAZ, 28KNAG, 28KNAL and 28KNAA). For the primary school the post codes of its catchment area were mounted onto computer and assigned to enumeration districts as above. All the enumeration districts were in the ward 28KNAZ and all but two of the enumeration districts in the ward were represented. Though the ward is certainly larger than the catchment area of the school it is difficult to estimate the population of the latter. Therefore the whole ward has been used to provide both numerators and population denominators.

Once estimates of the population denominators were available, observed and expected incidences were compared using the Poisson distribution. For point sources the "traditional" method is to compare observed $(O)$ and expected $(E)$ incidence in an area within a specified distance of the point. Such an area can be defined using wards or enumeration districts. So as to have increased flexibility we chose to use enumeration districts. The stack at Capper Pass is $183 \mathrm{~m}$ high and the factory management have informed us that maximum ground emission levels would be at a distance of 10 times the stack height. Therefore we delineated an area of investigation of up to 18.3 $\mathrm{km}$ from the stack (ie, up to 10 times the distance to maximum pollution levels). There are 766 enumeration districts in North Humberside with centroids $<18.3 \mathrm{~km}$ from the stack. Therefore values of $O$ and $E$ can be compared for 766 "circles". The difficulty encountered by studies of the traditional type is the choice of radius. Typically certain radii will show significant

*The centroid of an area represents a central point with due account being taken of population; it is specified for each area by the OPCS 
excesses while others do not. The first stage of our investigations was to examine each of the circles individually to see whether $O$ was significantly in excess of $E$. If there were none then this was taken as evidence that there was no association of risk with distance from the point. When, however, some individual values of $O$ were significantly higher than $\mathrm{E}$ we uscd the conditional Poisson maximum method of Stone. ${ }^{13-15}$ This method tests the largest ratio $\mathrm{O} / \mathrm{E}$, with adjustments for the fact that multiple tests have been made.

No attempt was made to examine aetiological hypothesis relating to clustering elsewhere in North Humberside since none were available. However the recommendations for future studies apply for the most part to the entire area.

Finally we reassessed the incidence in Hull 10 itself. For this a data dependent choice of adding the extra diagnostic subgroup of tumours of the central nervous system was included, this being the second largest group of childhood malignancies. Because of the status of this area as the site of the original anecdotal cluster and because of the data dependency we have adopted the conservative approach of computing the probability that any one of the 89 wards should have had an excess similar to the highest observed in any one of the wards in Hull 10.

\section{Results}

Table I shows the numbers of cases of childhood malignancy in the study area and in the rest of Yorkshire. The overall incidence rates in the two regions are almost identical though there is higher leukaemia incidence in North Humberside. Corresponding figures for the health districts are also included.

The Cuzick T statistics are shown in table II. Statistically significant evidence of spatial clustering for all malignancies and also for leukaemias is present. There is no evidence of clustering of the "non-leukaemias" so it is clear that the leukaemia distribution contributes substantially to the conclusion for "all malignancies". A similar analysis for the whole of Yorkshire showed no evidence of clustering. The locations of those cases which contribute most to the value of this statistic are shown in table III and the clustering is particularly strong in HU10 though there is no evidence that it is confined to

Table I Incidence of childhood malignancy 1974-1986. Rates per $10^{5}$ patient years are shown in parentheses.

\begin{tabular}{lllll}
\hline & \multicolumn{2}{l}{ Leukaemias } & Other malignancies & \multicolumn{1}{l}{ Total } \\
\hline N Humberside & $3.94(53)$ & $6.76(91)$ & $10.69(144)$ \\
Rest of Yorkshire & $3.25(271)$ & $7.48(624)$ & $10.73(895)$ \\
Beverley District (28KN) & $2.54(7)$ & $7.99(22)$ & $10.05(29)$ \\
East Yorkshire District $(28 \mathrm{KK})$ & $5.42(10)$ & $8.67(16)$ & $14.09(26)$ \\
Holderness District (28KU) & $1.56(2)$ & $5.47(7)$ & $7.03(9)$ \\
Kingston-upon-Hull (28KW) & $4.48(34)$ & $6.07(46)$ & $10.55(80)$ \\
\hline
\end{tabular}

Table II The Cuzick $T_{3}$ statistics applied to the Yorkshire Regional Childhood Tumour Register data for $N$ Humberside.

\begin{tabular}{lllll}
\hline Disease(s) & Observed value & Expected value & SD & p \\
\hline All malignancies & 242 & 217.5 & 13.3 & 0.03 \\
Leukaemias & 60 & 42.8 & 6.6 & 0.004 \\
Other malignancies & 116 & 106.4 & 9.2 & 0.15 \\
\hline
\end{tabular}

${ }^{a_{T}} \mathrm{~T}_{3}$ is a count of case-case links as one of the three nearest neighbouring addresses.
Table III Locations of influential cases for Cuzick's $T_{3}$ number of influential cases.

\begin{tabular}{lll}
\hline Post code sector & All malignancies & Leukaemias \\
\hline HU3 & 2 & 3 \\
HU4 & 1 & 0 \\
HU5 & 3 & 0 \\
HU6 & 1 & 0 \\
HU7 & 2 & 0 \\
HU8 & 0 & 1 \\
HU9 & 3 & 3 \\
HU10 & 7 & 4 \\
YO4 & 6 & 2 \\
YO15 & 2 & 1 \\
YO16 & 2 & 2 \\
YO25 & 1 & 0
\end{tabular}

${ }^{a}$ An influential case is one with a contribution of 2 (for leukaemias) and 3 (for all malignancies) to $T_{3}$; the difference is because of the differing case/control ratios.

that area. Figure 2 shows the spatial distribution of intensity of leukaemia incidence.

Observed and expected counts of wards with numbers of cases significantly in excess of expected figures according to the Poisson distribution for North Humberside and for the whole of Yorkshire are shown in table IV. This gives further evidence of non-uniformity of disease intensity of leukaemias in North Humberside which is not apparent in the whole of Yorkshire. No similar discrepancy is evident for the whole range of childhood malignancies. Similar results are obtained from an examination of ward case counts (table V).

The four putative aetiological associations for HU10 were then examined in turn.

Table VI gives comparisons of observed and expected incidence in the area of Wolfreton Secondary school and Poisson probabilities. There are indeed certain excesses, but there is no evidence of any excess in children of secondary school age.

Three leukaemias and one solid tumour (a retinoblastoma) were observed from the catchment area of Carr Lane Primary school. All of these were aged $<10$ years at diagnosis. Observed and expected values by disease and age group are also shown in table $\mathrm{V}$. There is an excess of leukaemias amongst young children in the area. However only one of the leukaemia cases was of primary school age at diagnosis. The other two were aged 2 and 4 respectively.

Cumulative observed and expected numbers were computed and compared for each "circle" comprising all enumeration districts with centroid less than a given distance from the stack at Capper Pass. The ratios of $\mathrm{O} / \mathrm{E}$ plotted against radius are shown in fig 3 . For the leukaemias there is no radius at which the difference between observed and expected numbers approaches statistical significance. For the other malignancies excesses are apparent both very close to the stack and approximately $7 \mathrm{~km}$ from it. For the latter group, Stone's Conditional Poisson maximum statistic is $13.75(p=0.01)$. However this result relies heavily on two cases in circles with small populations close to the factory. These two cases formed no part of the original "anecdotal" cluster. If instead of considering all circles only those with a child population $>1000$ are examined then the statistic becomes $1.95(p=0 \cdot 11)$.

The fourth putative source is located very close to Capper Pass and it might be expected that all the foregoing analysis could apply equally well to 
Figure 2 Localised clustering of disease in North Humberside. Intensity of shading represents intensity of case clustering; individual cases are not shown.

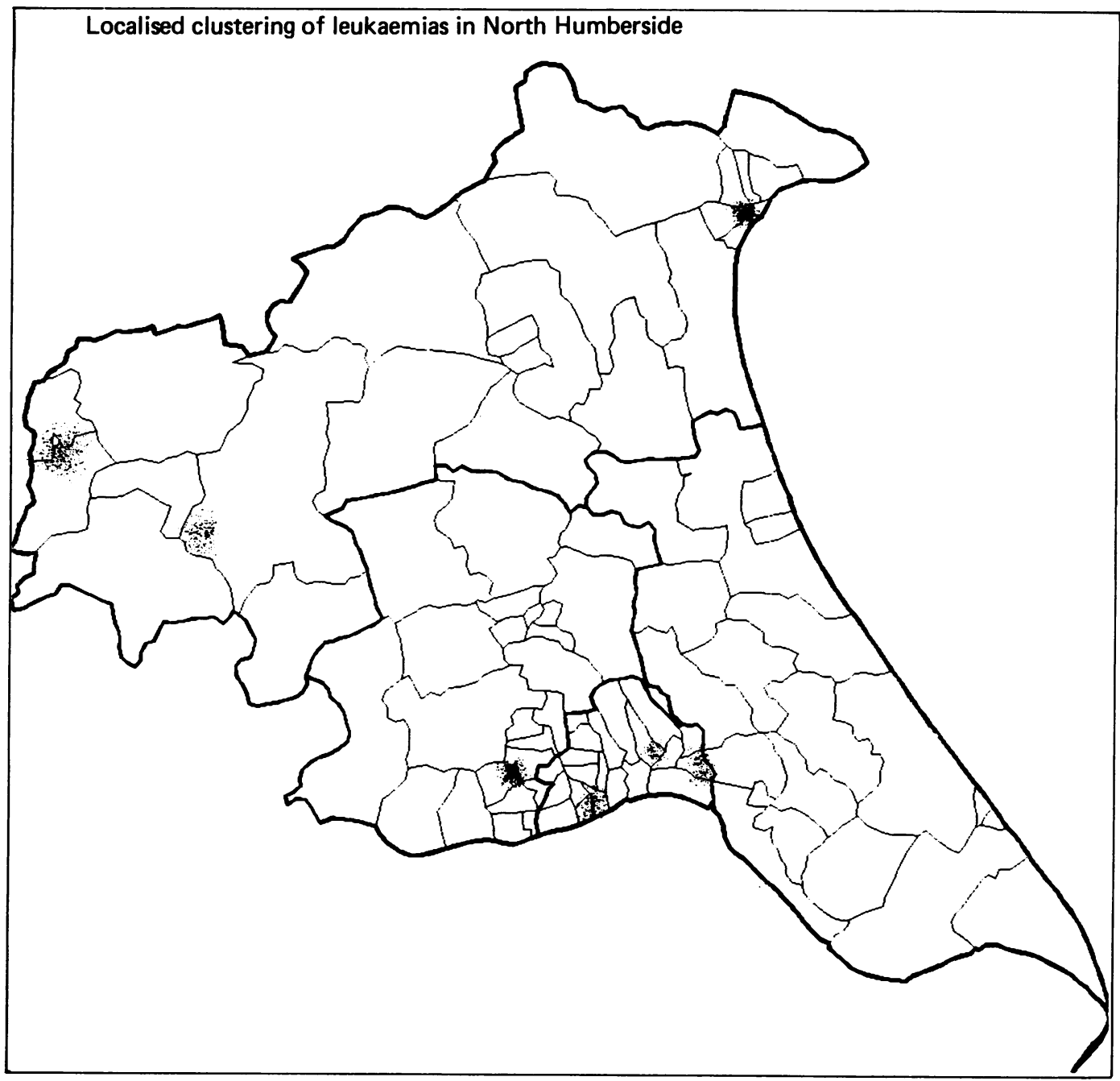

it. This is true of the negative result of the leukaemias; for the other malignancies Stone's conditional Poisson maximum statistic is 2.12 and this is not statistically significant $(p=0 \cdot 46)$.

A full list of Yorkshire Regional Childhood Tumour Register cases within $9.15 \mathrm{~km}$ of the stack at Capper Pass, ie, up to half the maximum distance we have considered, is given in table VII. This includes cases in the catchment area of either school and those outside the time period of the formal analysis. It is interesting that the cases who lived close to the factory at diagnosis (ie, closer than $6 \mathrm{~km}$ ) were all diagnosed before 1980 .

Examination of the diagnoses of the "clustered" cases which were identified in Hull 10 and of the list in table VI suggested that there might be an excess of central nervous system tumours. They also indicated that the unusual features of the distribution in Hull 10 were a local

Table IV The fit of the Poisson distribution to ward incidence data. Values are numbers of wards with excesses significant at the $5 \%$ level.

\begin{tabular}{|c|c|c|c|}
\hline & North Humberside & Rest of Yorkshire & Yorkshire \\
\hline $\begin{array}{l}\text { Observed } \\
\text { Expected }^{\mathrm{a}}\end{array}$ & $\begin{array}{l}6 \\
1 \cdot 83^{*}\end{array}$ & $\begin{array}{c}\text { aemias } \\
10 \\
8.93\end{array}$ & $\begin{array}{l}16 \\
10 \cdot 76\end{array}$ \\
\hline $\begin{array}{l}\text { Observed } \\
\text { Expected }\end{array}$ & All mc & $\begin{array}{c}\text { ignancies } \\
7 \\
12 \cdot 25\end{array}$ & $\begin{array}{l}10 \\
14 \cdot 66\end{array}$ \\
\hline
\end{tabular}

aexpected numbers are computed by calculating the exact probability for each ward and then summing; regional incidence rates are used throughout.
«statistically significant $(p=0.01)$ aggregation of leukaemias in one ward (28KNAZ), and a local aggregation of central nervous system tumours in another ward (28KNAG).

The first of these appears to be part of the generalised clustering of leukaemias in North Humberside. The leukaemia figures for 28KNAZ are $0=3, E=0.48$. This is unusual $(p=0.013)$ but

Table $V$ The fit of the Poisson distribution to ward incidence data. Values are numbers of wards with specified case counts. (observed number: expected number).

\begin{tabular}{|c|c|c|}
\hline Cases & Leukaemias & All malignancies \\
\hline $\begin{array}{r}0 \\
1 \\
2 \\
3 \\
4 \\
>5\end{array}$ & $\begin{array}{l}N \text { Humberside cases } \\
64: 58 \cdot 55 \\
12: 20.82 \\
5: 6 \cdot 39 \\
8: 3 \cdot 24\end{array}$ & 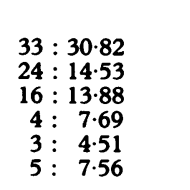 \\
\hline$\chi^{2}(\mathrm{df})$ & $11 \cdot 34(3)^{\star}$ & 3.63(5) \\
\hline $\begin{array}{r}0 \\
1 \\
2 \\
3 \\
4 \\
5 \\
6 \\
7 \\
8 \\
>9\end{array}$ & $\begin{array}{c}\text { Yorkshire cases } \\
346: 355.48 \\
108: 123.31 \\
52: 46.99 \\
17: 19.23 \\
8: \quad 7.37 \\
5: 3.61\end{array}$ & 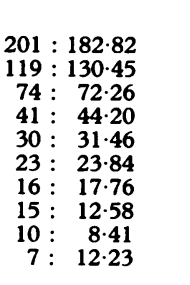 \\
\hline$\chi^{2}(\mathrm{df})$ & $3.61(5)$ & $6 \cdot 36(9)$ \\
\hline
\end{tabular}




\begin{tabular}{|c|c|c|c|}
\hline Age (population) & Leukaemias & Other malignancies & All diagnoses \\
\hline \multicolumn{4}{|c|}{ Wolfreton secondary school } \\
\hline \multirow{2}{*}{$\begin{array}{c}0-14 \\
(4848)\end{array}$} & $4 / 2 \cdot 05=1.95$ & $8 / 4 \cdot 66=1 \cdot 72$ & $12 / 6 \cdot 71=1 \cdot 79$ \\
\hline & $(p=0 \cdot 15)$ & $(p=0 \cdot 10)$ & $(p=0.04)$ \\
\hline \multirow{2}{*}{$\frac{11-14^{b}}{(1880)}$} & $1 / 0.35=2.86$ & $1 / 1 \cdot 27=0.79$ & $2 / 1 \cdot 61=1 \cdot 24$ \\
\hline & $(p=0.30)$ & $(p=0.64)$ & $(p=0.48)$ \\
\hline \multicolumn{4}{|c|}{ Carr Lane primary school ${ }^{a}$} \\
\hline \multirow{2}{*}{$\begin{array}{c}0-14 \\
(1189)\end{array}$} & $3 / 0 \cdot 48=6.25$ & $1 / 1 \cdot 13=0 \cdot 88$ & $4 / 1 \cdot 61=2 \cdot 48$ \\
\hline & $(p=0.01)$ & $(0=0.69)$ & $(p=0.09)$ \\
\hline \multirow{2}{*}{$\begin{array}{l}0-10^{b} \\
(728)\end{array}$} & $3 / 0 \cdot 40=7 \cdot 50$ & $1 / 0 \cdot 82=1.22$ & $4 / 1 \cdot 22=3.28$ \\
\hline & $(p=0.01)$ & $(p=0.56)$ & $(p=0.04)$ \\
\hline
\end{tabular}

apopulation denominators have been taken from the whole of ward 28KNAZ

b The population figures for these age groups and the expected incidence rates have been calculated by applying the Yorkshire age distribution to the small area populations.
Table VI Observed and expected incidence in the area of the schools $O / E$ and Poisson probabilities.

the probability that at least one of the wards in North Humberside would have an excess with Poisson $p$ value $<0.013$ is in fact 0.38 . Since we have shown that the distribution has more dispersion than that predicted by the Poisson distribution, this figure is too low.

The situation for central nervous system tumours in 28KNAG is different. A repeat of the analysis of Cuzick and Edwards shows no evidence of generalised clustering; there is no evidence of lack of fit of the Poisson distribution; however, the rates for North Humberside are considerably higher than those for Yorkshire (3.3/10 5 PY compared with $2 \cdot 35)$. The incidence figures for $28 \mathrm{KNAG}$ are $0=4, E=0.35$. This too is unusual $(p=0.0005)$ and the probability that at least one of the wards in North Humberside would have such an excess is 0.016 . Thus these malignancies appear to have a local incidence that may possibly be regarded as exceptional.

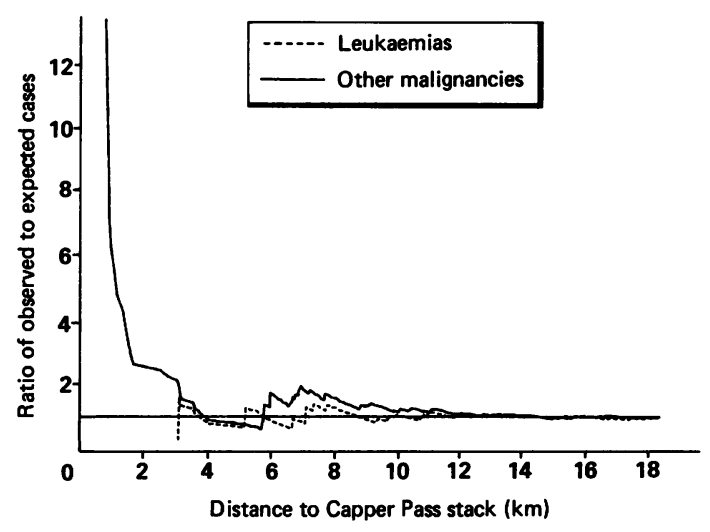

Ratios of expected incidence in relation to distance from Capper Pass Stack.

Table VII Cases from the Yorkshire Children's Tumour Registry-Resident <9.15 $\mathrm{km}$ from Caper Pass stack at diagnosis. This list contains all cases registered to August 1, 1988.

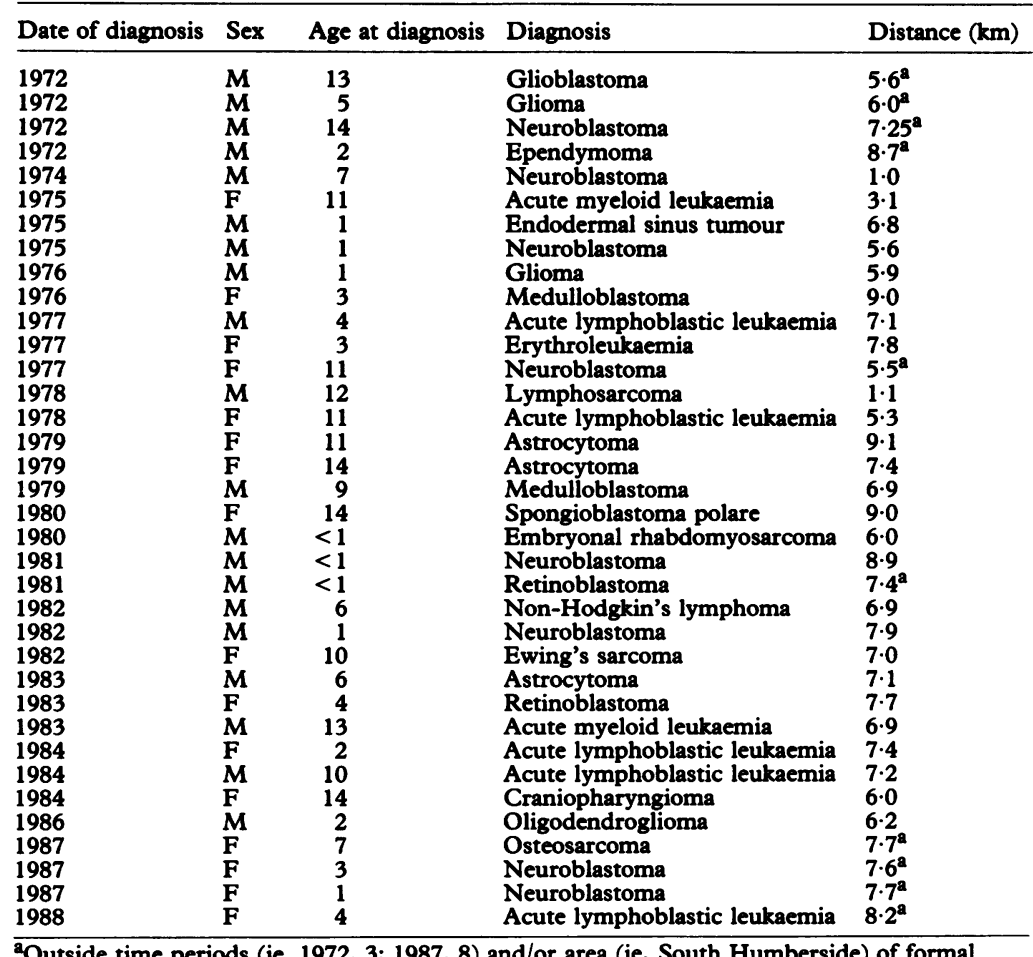

aOtside time periods (ie, 1972, 3; 1987, 8) and/or area (ie, South Humberside) of formal analysis.

\section{Discussion}

The investigation of spatial clustering of rare disease is extraordinarily difficult. One problem is the lack of uniformity of the spatial distribution of the underlying population and of precise local information about the distribution. Cuzick's method overcomes these difficulties provided only that the sampling frame used to obtain control addresses is satisfactory. There is no population register of children, but we have shown that the use of the birth register is suitable.

Two further problems arise because formal analysis is frequently conducted "post hoc" and liable to be influenced by biased overreporting of cases from anecdotal clusters and by data dependent choice of spatial, temporal or diagnostic criteria. The use of data from the Yorkshire Regional Childhood Tumour Registry should avoid the first problem since the method of case registrations has been uniform across the whole of Yorkshire. The temporal and diagnostic criteria are free of data dependence; the longest time period available for analysis has been selected and natural diagnostic classifications adopted.

The possible data dependence of the spatial boundaries in any investigation of an area which contains an "anecdotal" cluster raises complex issues. Nevertheless, because of high levels of public awareness statisticians and epidemiologists will continue to be confronted by the need to conduct post hoc analyses. The approach that we have taken here is to begin with an analysis of the spatial distribution of disease in a larger and natural geographical area; this includes the four health districts which contain the site of local concern but are not dominated by it. The method of Cuzick and Edwards has enabled us to do this independently of administrative boundaries. It has been shown that the distribution, particularly of the leukaemias, is unusual in Northern Humberside. There is statistically significant evidence of spatial clustering in the region, with clustering present at, but not confined to, the area of the anecdotal cluster. There is on the other hand no evidence that the distribution over the rest of Yorkshire is not uniform. To interpret the aetiological, as opposed to statistical, significance 
of this spatial pattern is difficult at present because relatively little is known about the "usual" spatial distribution elsewhere. However, Urquhurt et al (reported in ${ }^{16}$ ) have shown by a different method that the distribution of childhood leukaemias in Scotland appears to be uniform. A systematic study of the spatial distribution of these and related disease is currently in progress at the Leukaemia Research Fund Centre.

Although the original report of Cuzick and Edwards did not discuss the identification or display of clustering we have shown that both are possible. We are thus able to identify the locations of a number of clustered cases and also map intensity of clustering. Care is required when interpreting such clustering in terms of specific aetiological hypotheses. Commonly one particular favoured interpretation is put forward to explain case aggregations (eg ${ }^{4-6}$ ). In this instance, at least four were suggested, so it was possible to examine each in turn. Excesses in the catchment areas of the two schools have been shown. For the secondary school the case excess is concentrated in younger children and in solid tumours, and for the primary school an excess of leukaemias is present. In the latter instance two of the children were aged 4 at diagnosis and one aged 2. Evidence directly involving either school is not supported by our findings unless pupil-sibling contacts are involved.

Capper Pass factory is licensed to emit small quantities of ${ }^{210}$ polonium. Like all smelters it will also emit a variety of heavy metals, including arsenic, lead and zinc, in small but quantifiable amounts. A tentative relationship between incidence rates and distances from the factory has been established for malignancies other than leukaemias. However our findings do not support a hypothesis relating leukaemia incidence to distance from the stack. Thus pollution from the Capper Pass stack is unlikely to be associated with the leukaemia clustering, at least in a unifactorial way, although there is a possibility of its involvement in the aetiology of the other malignancies. This difference is very interesting because media attention has been focused on radiation, an environmental hazard linked particularly to leukaemogenesis and not to other childhood malignancies. ${ }^{5}$

The methods used in considering the four putative associations can be criticised. For example, the presence of generalised spatial clustering is a contraindication to the use of the Poisson distribution and this applies especially to the leukaemias. An alternative distribution would have higher dispersion. Thus it is important to note that the conclusions for the leukaemias are essentially negative-a conclusion which would be upheld if using an alternative distribution. Where tentative positive conclusions have been based on the Poisson distribution it has been for the non-leukaemias and central nervous system tumours for which no evidence of a nonPoissonian distribution has been found. The method of assigning cases to enumeration districts, while the best available from the England and Wales 1981 census, is not ideal. Moreover weather conditions and particularly the direction of prevailing winds have not been considered. It is clear, however, that no methodological refinements applied to these data would enable a firm conclusion of causality for any one of the putative factors. Certain causal links have been refuted. We believe that this study unequivocally shows the need for further detailed investigations in North Humberside. The aetiologies of childhood leukaemias and other childhood malignancies are largely unknown and an area with an unusual spatial distribution is likely to increase our understanding of these diseases.

To investigate a hypothesis of social contact (especially school) linkage, a case-control study is essential. This should concentrate on social links of children and their siblings and use the design of Pike and Smith. ${ }^{17}$ To examine the possibility of a localised non-infective aetiological agent, the adult incidence of leukaemias, lymphomas and brain tumours in the same area is being studied. In order to relate this incidence to industrial pollution it is the intention that radiation and other environmental pollutants can be systematically measured over a grid. In the case of radiation this study is already in progress. Further studies of an effect related to radiation and specific to children should include an examination of incidence rates around other sites in Yorkshire licensed to emit ${ }^{210} \mathrm{Po}$. Biological samples should be collected from new cases arising in the relevant area for virological and other investigations. Until these investigations are completed, it is unlikely that any aetiological conclusions will be appropriate.

Finally, so far as methods are concerned we conclude that the test of Cuzick and Edwards can and should be used as a first step in the investigation of a reported "cluster". This will describe the general spatial distribution of disease in the vicinity and it will then be possible to assess the local aggregation. Essentially there are three possible conclusions; the distribution in the broad area may be unusual and the reported cluster a manifestation of this, the distribution in the broad area may be uniform but the local aggregation may indeed be exceptional, or the entire spatial pattern may be consistent with uniform risk. It would appear that leukaemias and central nervous system tumours may be examples of each of the firsi two situations. Geographical investigations are very unlikely to result in the identification of a "cause". Instead many laborious and expensive studies will be required.

We are indebted to Dr Jack Cuzick whose method we have used. Our contact with Drs Stone and Bithell is also gratefully acknowledged. We thank Drs Michael Hills, David Clayton and Peter Boyle for discussions which have contributed to the final form of this article.

Financial support from Leeds Candlelighters and Leeds Western Special Trustees for the Yorkshire Childrens Tumour Registry is gratefully acknowledged, as is the assistance of Dr C C Bailey, Dr S C Cartwright, Dr I Lewis and numerous other consultants within the Yorkshire region. We thank Professor $\mathrm{C}$ Joslin and the Yorkshire Regional Cancer Registry for supplying data so readily. Mr J Miller's help with data was invaluable. Mr J Ricketts developed the mapping software which has been used here. Finally we would thank Mr Jon Dunnington for assistance with data processing and Miss Lorraine Harvey for typing the manuscript. The Leukaemia Research Fund support the Clinical 
Epidemiology Unit where the work presented was undertaken. Digitised boundary information and other related material remain the property and copyright of the Crown.

\section{Appendix}

DISPLAYING THE LOCAL INTENSITY OF DISEASE The Cuzick-Edwards method makes no provision for display; the following procedures gives a method which for simplicity we give for $k=3$.

For any case, located at a point $P$, its contribution, $\theta$, to the $T_{3}$ statistic is one of the values $0,1,2,3$. These values can then be interpreted as ranks for the local intensity of disease at $\mathbf{P}$ with $\theta=3$ indicating high intensity and $\theta=2$ moderate intensity (see the influential points in table III). This computation has involved some averaging or smoothing over a circle whose radius is the distance, $r$, from the case to its third nearest neighbour.

We can display high or moderate intensity by shading the circle centre $P$ and radius $r$ with depth of shading depending on $\theta, r$ and distance from $P$. This could be done in a variety of ways but we have adopted the following procedure which allocates a "probability of plotting" to each individual pixel in the circle. This probability takes maximum value at $P\left(1\right.$ if $\theta=3$ and $\frac{1}{2}$ if $\theta=2$ ) and then decreases slowly at first then more rapidly to 0 at the circumference. Where two or more circles overlap the probability of plotting is calculated for each circle and the maximum used. For technical reasons a minimum circle size is allocated; this minimum is a map area rather than a geographical area.

The display produced in this way shows intensity of clustering by darkness of shading; the size of a shaded area indicates only the density of the population. To be able to use the method to illustrate the spatial pattern for more than one data set in such a way that they can be compared it is necessary to fix both $\mathrm{k}$ and the number of controls/case.
1 Cartwright RA, Alexander FE, Carrette J, et al. Leukaemia and lymphoma distribution in parts of the United Kingdom during 1984 and 1985. Leukaemia Research Fund Centre 1987 (Unpublished report)

2 Cartwright RA, McKinney PA. Childhood cancer and adult malignancies in the Hull 10 postal area of East Yorkshire Health District. Leukaemia Research Fund 1985. Report submitted to District Medical Officer of East Yorkshire. 3 Smith PG. Current assessment of "case-clustering" of

4 Linet MS. The leukaemias: epidemiological aspects. Oxford: Oxford University Press, 1985.

5 Black D. Investigation of the possible increased incidence of cancer in West Cumbria. Report of the Independent cancer in West Cumbria. Report of the
Advisory Group. London: HMSO, 1984.

6 Heasman MA, Urquhurt JD, Black RI, Kemp IW. Leukaemias in young persons in Scotland: a study of its Leukaemias in young persons in Scotland: a study of its geographical distribution and relationship to
installations. Scottish Health Bull 1987; 45, No 3.

7 Lagakos SW, Wessen BJ, Zelen M. An analysis of contaminated well water and health effects in Wobura, Massachusetts. F Am Stat Assoc 1986; 395: 583-96.

$8 \mathrm{Knox}$ EG. The detection of space-time interactions. Appl Stat 1964; 18: 17-24.

9 Chen $R$, Mantel N, Klingberg MA. A study of the techniques for space-time clustering in Hodgkin's disease. Stat Med 1984; 3: 173-84.

10 Cuzick J, Edwards R. Spatial clustering for inhomogeneous populations. I R Stat Soc 1989 (in press).

11 Alexander FE, Cartwright RA, McKinney PA. Childhood malignancies in West Hull. Lancet 1988; i: 769, and correction 896.

12 Birch JM, Marsden HB. A classification scheme for childhood cancer. Int $f$ Cancer 1987; 40: 620-4.

13 Stone RA. Statistical methodology and causal inference in studies of the health effects of radiation. Oxford: Oxford University, 1986. $\mathrm{PhD}$ thesis.

14 Stone RA. Investigations of excess environmental risks around putative sources: Statistical problems and a proposed test. Stat Med 1988; 7: 649-60.

15 Bithell JF, Stone RA. On statistical methods for analysing the geographical distribution of cancer cases near nuclear installations. $\mathcal{f}$ Epidemiol Community Health 1989; 43: 79-85.

16 Committee on Medical Aspects of Radiation in the Environment (COMARE). Investigation of the possible increased incidence of leukaemia in young persons near the Dounreay Nuclear Establishment, Caithness, Scotland. London: OPCS, 1988.

17 Pike MC, Smith PG. A case-control approach to examine diseases for evidence of contagion. Biometrics 1974; 24: 263-79. 